\title{
Nonlinear Finite Element Analysis of Structures Strengthened with Carbon Fibre Reinforced Polymer: A Comparison Study
}

\author{
X.S. Yang, J.M. Lees, and C.T. Morley \\ Department of Engineering, University of Cambridge \\ Trumpington Street, Cambridge CB2 1PZ, UK \\ xy227@eng. cam. ac.uk
}

\begin{abstract}
Modelling crack propagation in fracture mechanics is a very challenging task. Different methods are usually robust under different conditions and there is no universally efficient numerical method for dynamic fracture simulations. Most available methods are computationally extensive and usually require frequent remeshing. This comparison study focuses on three major methods: the discrete element method, the adaptive fixed crack method and the element-free Galerkin method. By implementing these methods to study a $2 \mathrm{D}$ concrete beam with reinforcement of carbon-fibre reinforced polymer straps, we have shown that for simulations of a limited number of cracks, fixed crack method gives the best results. For multiple crossover cracks, the discrete element method is more suitable, while for moderate number of elements, the element-free Galerkin method are superior. However, for large number of elements, fixed crack method is most efficient. Comparisons will be given in details. In addition, new algorithms are still highly needed for the efficient simulations of dynamic crack propagations.
\end{abstract}

\section{Introduction}

The technique of strengthening reinforced concrete structure using carbon fibrereinforced polymer (CFRP) is promising and can have important applications in many areas. However, an understanding of the behaviour and influence of the FRP composite system is crucial for the proper design of structural reinforcement strategies in infrastructure such as bridges and buildings 6, 7]. The computer simulation is an cost-effective way of improving such an understanding.

In simulating the fracture and crack growth, there are three major paradigms: discrete element methods, smeared crack methods and element-free Galerkin methods. The discrete element method has the advantage of tracing each individual crack with an irregular geometry. In order to do this, substantial remeshing is required to accommodate the arbitrary geometry and boundaries along the newly created cracks and thus extensive computation is required which is usually time-consuming even with the modern fast computers. The adaptive fixed crack concept or smeared crack model is very efficient in computation but cannot directly deal with the crack propagation, especially in the case of multiple cracks. 
This method uses the equivalent material properties to simulate the effect of the cracks in the context of continuum-based finite element analysis [4,5. On the other hand, the new promising element-free Galerkin method has the ability of dealing with an arbitrary geometry but it is still computationally extensive compared with the conventional finite element methods, especially in the interface regions and crack zones [1, 10, 11]).

In this paper, we will compare different numerical approaches to the nonlinear finite element simulations of concrete structures with CFRP reinforcement and analyse the appropriate conditions in detail. The main focus is to find an appropriate way to simulate the nonlinear behaviour of concrete beams strengthened with CRFP straps. In addition, we will also discuss the implication of simulation paradigms in engineering applications.

\section{Models and Their Formulations}

In order to compare different numerical methods, we first outline their formulations briefly, and then we implement them to simulate the same $2 \mathrm{D}$ concrete beam with CFRP reinforcement.

\subsection{Discrete Element Method}

The discrete element method for rigid granular materials was developed by Cundall and Strack [3] and this method was later incorporated into a combined finite-discrete element method [9]. There are some variations of this method, and the main procedure takes the whole domain as a multibody system and each domain is considered as a finite element continuum where the usual finite element analysis applies. The total strain increment $\varepsilon_{i j}$ consists of the elastic strain $\varepsilon_{i j}^{e}$ and the inelastic strain $\varepsilon_{i j}^{p}$, or

$$
\varepsilon_{i j}=\varepsilon_{i j}^{e}+\varepsilon_{i j}^{p}
$$

The general stress-strain relation and strain energy density $U$ are

$$
\sigma_{i j}=C_{i j k l} \varepsilon_{k l}, \quad U=\frac{1}{2} C_{i j k l} \varepsilon_{i j} \varepsilon_{k l}
$$

where the elastic constants satisfy certain symmetry conditions such as $C_{i j k l}=$ $C_{k l i j}=C_{i j l k}$ and $i, j=1,2,3$. The interactions between different domains will be computed using Hertz kinetics.

\subsection{Smeared Crack Model}

In the smeared crack model, the total strain increment is decomposed into a concrete strain increment $d \varepsilon^{c o}$ and a crack/fracture strain increment $d \varepsilon^{f}$, in a similar fashion as the decomposition of the total strain into an elastic strain increment and a plastic strain increment in elasto-plastic formulation. One can 
further decompose the concrete strain increment into an elastic part $d \varepsilon^{e}$ and a plastic part $d \varepsilon^{p}$. Thus, the decomposition becomes

$$
d \varepsilon_{i j}=d \varepsilon_{i j}^{e}+d \varepsilon_{i j}^{p}+d \varepsilon_{i j}^{f} .
$$

The transformation is necessary from the local coordinate system de which is aligned with the crack to the global coordinate system $(\mathbf{d} \varepsilon)$. We use the same conventional notation as de Borst [4] and Kesse [8] where the subscript $n$ means normal to the local crack plane and $t$ means tangent to the crack plane. After some tedious but straightforward calculations, we have

$$
\mathbf{d} \sigma=\left[\mathbf{I}-\mathbf{N}\left(\mathbf{D}^{\mathbf{c r}}+\mathbf{N}^{\mathbf{T}} \mathbf{D N}\right)^{-\mathbf{1}} \mathbf{N}^{\mathbf{T}}\right] \mathbf{D d} \varepsilon .
$$

The matrix $\mathbf{D}^{\mathbf{c r}}$ can be considered as a normal crack stiffness $\mathbf{D}_{\mathbf{n}}^{\mathbf{c r}}$ and a shear crack stiffness $\mathbf{D}_{\mathbf{t}}^{\mathrm{cr}}$, i.e.,

$$
\mathbf{D}^{\mathbf{c r}}=\mathbf{D}_{\mathbf{n}}^{\mathbf{c r}}+\mathbf{D}_{\mathbf{t}}^{\mathbf{c r}}
$$

where

$$
\mathbf{D}_{\mathbf{n}}^{\mathbf{c r}}=\left(\begin{array}{cc}
k_{c} & 0 \\
0 & 0
\end{array}\right), \quad \mathbf{D}_{\mathbf{t}}^{\mathbf{c r}}=\left(\begin{array}{cc}
k_{n n} & k_{n t} \\
k_{t n} & k_{t t}
\end{array}\right),
$$

where $k_{c}, k_{n n}$ etc are the constants determined from experiments. It is worth pointing out that the choice of the values for these parameters is relatively arbitrary and detailed studies are highly needed.

\subsection{Element-Free Galerkin Method}

The element-free Galerkin method was developed by Belytschko and his colleagues [1,2, and a coupled finite element-element-free Galerkin (EFG) method was applied by Sukumar et al to simulate a fracture problem [10. The main advantage of an EFG method is that it requires only nodal data for constructing approximate functions and no element structure is necessary. In this sense, it is not a method based on interpolants but a method using moving least square approximations. The moving least square approximations are constructed in the following manner. Let the $m$-term approximation be

$$
\hat{u}(\mathbf{x}) \equiv \mathbf{p}^{\mathbf{T}}(\mathbf{x}) \mathbf{a}(\mathbf{x})=\sum_{j=1}^{m} p_{j}(\mathbf{x}) a_{j}(\mathbf{x}),
$$

where $p_{j}(\mathbf{x})$ are basis functions. The associated coefficients $a_{j}(\mathbf{x})$ in the approximation are determined by minimizing the quadratic form $\Phi(\mathbf{x})$

$$
\Phi(\mathbf{x})=\sum_{i}^{n} w_{i}(\mathbf{x})\left[\mathbf{p}^{\mathbf{T}}(\mathbf{x}) \mathbf{a}(\mathbf{x})-u_{i}\right]^{2}, \quad w_{I}(\mathbf{x})=w\left(\mathbf{x}-\mathbf{x}_{i}\right) \geq 0,
$$

where $w_{I}$ is a weighting function and $u_{i}$ is a nodal parameter. This is equivalent to define a EFG shape function

$$
\phi_{i}(\mathbf{x})=\sum_{j}^{m} p_{j}(\mathbf{x})[\mathbf{Q}]_{j i}
$$

where $\mathbf{Q}$ is a matrix function of $\mathbf{w}_{\mathbf{i}}(\mathbf{x})$ and $\mathbf{p}\left(\mathbf{x}_{\mathbf{i}}\right)$. It is worth pointing out that $u_{i} \neq \hat{u}\left(x_{i}\right)$ as $\hat{u}(\mathbf{x})$ is only an approximant. 


\section{$3 \quad$ Simulations and Results}

By implementing these three major numerical methods, we can now start to simulate crack propagation for various structures with CFRP reinforcement. For simplicity of comparison, we use the same fundamental structure of a $2 \mathrm{D}$ concrete beam for all simulations.

\subsection{Discrete Element Method: Fracture of Concrete Beam}

In order to demonstrate the simulation capability of the discrete element method, we first show the fracturing of a concrete beam due to an impact of an elastic block moving downward with an initial velocity $5 \mathrm{~m} / \mathrm{s}$. The dimensions for the beam are $2200 \mathrm{~mm}$ (length), 200mm (width) and 100mm (thickness). Figure 1 shows the different stages of the fracture patterns. The visualization has been carried out using Abaqus. The values used are $E=22 \mathrm{GPa}, \nu=0.2, \sigma_{Y}=$ $3.36 \mathrm{MPa}$, and $\rho=2400 \mathrm{~kg} / \mathrm{m}^{3}$. We can see that discrete element method is very powerful in fracture simulations and different fragments are computed in a realistic manner. Fracture patterns are traced as the crack propagates in each time step with local remeshing.
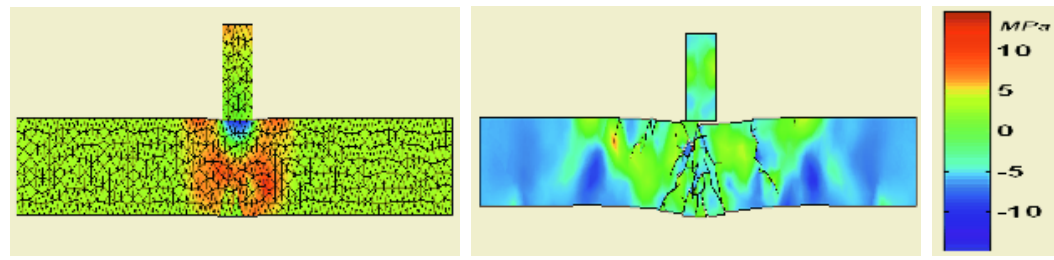

Fig. 1. The impact of a drop load on a $2 \mathrm{D}$ concrete beam and the fracture pattern at $t=0.1 \mathrm{~s}$ and $t=0.5 \mathrm{~s}$

\subsection{Adaptive Fixed Crack Model}

We now proceed to investigate the four-point bending of the same $2 \mathrm{D}$ concrete beam in the plane strain condition. The loading is applied as a linear function of time with a peak value of $f=100 \mathrm{kN}$, and the maximum time step $t=10,000$ (pseudo time). We can see from Figure 2 that the vertical cracks start to form and grow gradually as the loading force increases, and shear cracks start to

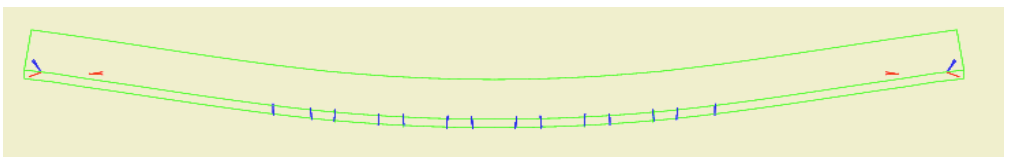

Fig. 2. The crack pattern for a $2 \mathrm{D}$ concrete beam with four-point bending conditions. Crack pattern are mainly vertical in the flexural section that is consistent with experimental results 
appear once the loading force reaches $40 \mathrm{kN}$. However, the crack pattern is just an indication and does not correspond to the real fracture although the overall patterns of the fracture are similar to that derived from experiments.

\subsection{Element-Free Galerkin Method}

A typical distribution of nodal points for the analysis of a $2 \mathrm{D}$ beam is shown in Figure 3. As no elements are requried in the element-free Galerkin method, only adaptive points are shown. The location of these points can be distributed in an adaptive manner where the density of the points varies with the potential density of cracks. The stress distribution $\sigma_{x x}$ at time $t=10,000 \mathrm{~s}$ is also shown. Red color corresponds to high shear stress and light green corresponds to compression. However, although the crack geometry are still limited by the density of meshless points, this method is much efficient compared to the discrete element method.
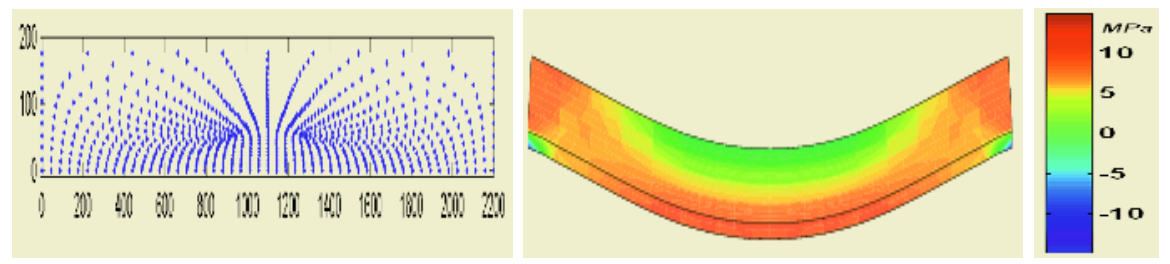

Fig. 3. Random points or locations for the EFG analysis, stress $\sigma_{x x}$ and the crack patterns at $t=10,000 \mathrm{~s}$ and $f=50 \mathrm{kN}$

\section{Conclusion}

By implementing three major numerical methods for simulating crack formation in a concrete beam, we have carried out a comparison study. A comprehensive comparison of three major methods for modelling the nonlinear behaviours of structures shows that for a limited number of cracks (less than 40) and elements $(<2000)$, the smeared crack model gives the best results. For multiple cracks (up to 100 cracks), the discrete element give the best results, while for moderate large number of elements with arbitrary geometry, the element- free Galerkin method seems superior. However, the number of cracks may be limited in this case. Simulations also suggest that for the choice of numerical methods shall take the type of problem into consideration even though material properties are the same. In order to get a sharp crack indication or trace multiple cracks, fine or dense meshes are recommended for all methods. In addition, the development of new efficient algorithms for fracture analysis is still highly needed.

\section{Acknowledgement}

The authors thank the financial support by EPSRC. 


\section{References}

1. T Belytschko, Y Y Lu, L Gu, Element-free Galerkin method, Int. J. Num. Meth. Eng., 37 (1994) 229-256.

2. T Belytschko, L Gu and Y Y Lu, Fracture and crack growth by element free Galerkin methods, Modelling Simul. Mater. Sci. Eng., 2 (1994) 519-534.

3. P A Cundall and O D L Strack, A discrete element model for granular assemblies, Geotechnique, 29, 47-65 (1979).

4. R de Borst, Smeared cracking, plastity, creep and thermal loading - a unified approach, Comp. Meth. Appl. Mech. Eng., 62 (1987) 89-110.

5. R de Borst and A H van den Boogaard, Finite element modeling of deformation and cracking in elary-age concrete, J. Eng. Mech. Div., ASCE 120 (1994) 2519-2534.

6. J M Lees, A U Winistoerfer and U Meier, External prestressed CFRP straps for the shear enhancement of concrete, ASCE J Composites for Construction, 6 (2002).

7. N A Hoult and J M Lees, Shear retrofitting of reinfoced concrete beams using CFRP straps, Proc. Advanced Composite Materials in Bridges and Structures, 2023 July, Calgary, (2004).

8. G Kesse, Concrete beams with external prestressed carbon FRP shear reinforcement, PhD Thesis, Cambridge University, (2003).

9. A Munjiza, D R J Owen, and N Bicanic, A combined finite-discrete element method in transient dynamics of fracturing solids, Engineering Computation, 12, 145-174 (1995).

10. N Sukumar, B Moran, T. Black, T Belytschko, An element-free Galerkin method for three-dimensional fracture mechanics, Computaional Mech., 20 (1997) 170-175.

11. Zienkiewicz O. C. and Taylor R. L., The Finite Element Method, Vol. I/II, McGrawHill, 4th Edition, 1991. 